\title{
Drosophila Double parked: a conserved, essential replication protein that colocalizes with the origin recognition complex and links DNA replication with mitosis and the down-regulation of $S$ phase transcripts
}

\author{
Allyson J. Whittaker, Irena Royzman, and Terry L. Orr-Weaver ${ }^{1}$ \\ Whitehead Institute and Department of Biology, Massachusetts Institute of Technology, Cambridge, Massachusetts 02142 USA
}

\begin{abstract}
We identified a Drosophila gene, double parked (dup), that is essential for DNA replication and belongs to a new family of replication proteins conserved from Schizosaccharomyces pombe to humans. Strong mutations in dup cause embryonic lethality, preceded by a failure to undergo $S$ phase during the postblastoderm divisions. dup is required also for DNA replication in the adult ovary, establishing that dup is needed for DNA replication at multiple stages of development. Strikingly, DUP protein colocalizes with the origin recognition complex to specific sites in the ovarian follicle cells. This suggests that DUP plays a direct role in DNA replication. The dup transcript is cell cycle regulated and is under the control of E2F and Cyclin E. Interestingly, dup mutant embryos fail both to downregulate $S$ phase genes and to engage a checkpoint preventing mitosis until completion of $S$ phase. This could be either because these events depend on progression of $S$ phase beyond the point blocked in the dup mutants or because DUP is needed directly for these feedback mechanisms.
\end{abstract}

[Key Words: DNA replication; amplification; cell cycle; Cdt1; E2F]

Received April 6, 2000; revised version accepted May 23, 2000.

Proper regulation of replication initiation is critical for maintaining the integrity of the genome. Many proteins required for replication initiation were first isolated genetically in Saccharomyces cerevisiae and Schizosaccharomyces pombe. Identification of sequences that comprise origins of replication permitted isolation of additional replication initiation proteins (for review, see Dutta and Bell 1997). Many of the proteins required for replication initiation are conserved between $S$. cerevisiae, $S$. pombe, and metazoans, implying that the mechanism of replication initiation is conserved (for reviews, see Dutta and Bell 1997; Donaldson and Blow 1999). The six member origin recognition complex (ORC) binds to the $S$. cerevisiae ARS element, a demonstrated origin of replication (Bell and Stillman 1992). The Cdc6 protein is recruited to the origin by ORC and in turn recruits the six member MCM complex to origins (Coleman et al. 1996; Donovan et al. 1997; Tanaka et al. 1997). Although MCMs are required for initiation, MCM 4,6, and 7 have been shown to travel with the elongation forks in $S$. cerevisiae. This observation, combined with the demon-

${ }^{1}$ Corresponding author.

E-MAIL weaver@wi.mit.edu; FAX (617) 258-9872. stration that human MCM proteins 4,6 and 7 and the single archaeon MCM protein have helicase activity in vitro suggests the MCM proteins have an additional role in elongation (Aparicio et al. 1997; Ishimi 1997; Kelman et al. 1999; Chong et al. 2000). In addition to cell-cycle control of the start of $S$ phase regulated by cyclin-dependent kinases (Cdks), origin activity is affected by the S phase specific Cdk, Cdc7/Dbf4 (Hartwell 1973, 1978; Hollingsworth and Sclafani 1990; Yoon and Campbell 1991; Jackson et al. 1993).

Although the mechanism of replication initiation appears to be conserved between $S$. cerevisiae, S. pombe and metazoans, replication origins are distinct. Origins of replication in $S$. cerevisiae, ARS elements, are relatively small (100-150 base pairs) and contain two essential regions, an A element that has an essential $11 \mathrm{bp}$ sequence referred to as the ACS (ARS consensus sequence) and several less-conserved B elements (Marahrens and Stillman 1992). In the fission yeast S. pombe, which is evolutionarily distant from $S$. cerevisiae, origins of replication are larger and more complex, 0.5-1 kb long. Several AT-rich regions have been found in $S$. pombe ARSs that are important for replication, but a true consensus similar to the ACS in S. cerevisiae has 
not been defined (Dubey et al. 1994; Clyne and Kelly 1995). Origins in metazoans have proven to be even more complex (for review, see DePamphilis 1999). The higher degree of complexity and flexibility may be required to contend with the changes in replication and transcriptional control that occur during metazoan development.

Drosophila provides a powerful model for understanding replication control in metazoans. The genetic tools available in Drosophila allow one to isolate mutations in both known and new replication proteins. Orthologs of ORC, MCMs, Dbf4, and Cdc6 are present in Drosophila, and many of these proteins have been shown to be necessary for proper replication (Feger et al. 1995; Gossen et al. 1995; Treisman et al. 1995; Su et al. 1996; Landis et al. 1997; Pak et al. 1997; Chesnokov et al. 1999; Landis and Tower 1999). Another advantage is that there are defined replicons (for reviews, see Orr-Weaver 1991; Royzman and Orr-Weaver 1998; Calvi and Spradling 1999). These replicons are responsible for amplification of four genomic intervals in the ovarian follicle cells, two of which produce the chorion proteins for the egg shell. Amplification is under developmental control, and cis-acting regulatory regions have been defined. In cytological studies ORC1 and ORC2 localize to these sites of amplification in the follicle cells, and ORC has been shown to bind to these amplification elements in vitro and in vivo (Asano and Wharton 1999; Austin et al. 1999; Royzman et al. 1999). Mutations in the orc2 gene or a dbf4-like gene reduce amplification (Landis et al. 1997; Landis and Tower 1999). These observations suggest that the mechanism of initiation used by these origins is similar to that used in genomic replication.

Embryogenesis is another developmental stage during which DNA replication and cell cycle control can be investigated in Drosophila (for review, see Foe et al. 1993). The first thirteen division cycles are controlled by maternal products and consist of $S$ phase followed by mitosis. At cycle 14 the cycles come under zygotic control with the introduction of a G2 phase during which transcription occurs. Cycles 14, 15, and 16, the postblastoderm cycles, occur in a stereotypic developmental pattern. In these cycles, $S$ phase immediately follows $M$, and transcripts for genes needed for $S$ phase are constitutively high (Duronio and O'Farrell 1994). After cycle 16 is completed, a G1 phase is added to the cell cycle, and $S$ phase transcripts are downregulated. At this time the larval tissues switch to an endo cycle where $S$ phase oscillates with a gap phase with no intervening mitoses (Smith and Orr-Weaver 1991). These S phases are preceded by a burst of transcription of $S$ phase genes under the control of the E2F transcription factor (Duronio et al. 1995, 1998; Royzman et al. 1997).

Through genetic studies in Drosophila, we identified a replication protein, the product of the double parked (dup) gene, that belongs to a new family of replication proteins. The DUP protein colocalizes with ORC2 at amplification foci, suggesting it is directly involved in DNA replication. In addition to its role in replication, dup mutations eliminate the checkpoint that makes mitosis dependent on S phase. This is reflected in the gene name: double parked was chosen because strong mutations in the gene block DNA replication during embryogenesis but nevertheless enter and arrest in mitosis, parking at two points in the cell cycle. Moreover, S phase transcripts are not downregulated in the mutants and remain constitutively high.

\section{Results}

Identification of a gene essential for DNA replication

We recovered four alleles of the dup gene in a screen for mutations that alter a G1/S transcriptional program during embryogenesis (Royzman et al. 1997). We identified a deficiency that uncovers dup, $D f(2 R) J P 1$ and found that a previously existing mutation, 1(2)51Ec (Underwood et al. 1990; Smith et al. 1993), is an allele of dup. All five alleles are embryonic lethal in trans to the deficiency. In addition to these strong dup mutations, the female-sterile mutation, $f_{S}(2) P A 77$, is an allele of dup. The dup $p^{a 1-a 4}$ mutations failed to complement $f_{S}(2) P A 77$; trans-heterozygotes had low viability and were female and male sterile. In contrast, 1(2)51Ec and $f_{s}(2) P A 77$ did complement and were both viable and fertile, thus they were previously thought to be separate genes (Underwood et al. 1990; Smith et al. 1993). The ability of these alleles to complement may be because 1(2)51Ec is a weaker allele than the other embryonic lethal mutations.

The dup mutants are defective in DNA replication both in embryogenesis and in oogenesis. To analyze DNA replication in dup mutants, embryos were isolated from females heterozygous for dup ${ }^{a 1}$ that had been crossed to heterozygous males and pulse labeled with bromodeoxyuridine (BrdU). Homozygous mutant embryos were distinguished from heterozygous embryos by using a marked balancer chromosome (see Materials and Methods). In the dup mutants DNA replication appeared to be normal through $S$ phase of cycle 15 . This is most likely because maternal pools of DUP protein suffice for the earlier embryonic replication cycles (data not shown). In contrast, BrdU incorporation was not detectable in cycle 16 (Fig. 1A,B). The block in replication in dup $^{a 1} /$ dup $^{a 1}$ homozygous mutant embryos occurs early in $S$ phase, because no BrdU incorporation was seen in the nuclei.

In addition to its role in embryogenesis, the $f_{S}(2) P A 77$ mutation demonstrates that dup is necessary for DNA replication in adult tissues. Homozygous mutant $f_{S}(2) P A 77$ females lay eggs with thin egg shells, and chorion amplification levels are decreased (Underwood et al. 1990). Follicle cell genomic replication and amplification can be visualized directly by BrdU labeling of ovaries (Calvi et al. 1998). Between stage 9 and early stage $10 \mathrm{~A}$ genomic replication ceases, and BrdU incorporation is no longer detectable throughout the nucleus. In stage $10 \mathrm{~B}, \mathrm{BrdU}$ is observed at four specific foci that are sites of amplification (Fig. 1C). Previous BrdU incorporation studies showed that chorion amplification is decreased uniformly in the follicle cells of $f_{S}(2) P A 77$ homozygous mutant females (Calvi et al. 1998). Similarly, we ob- 

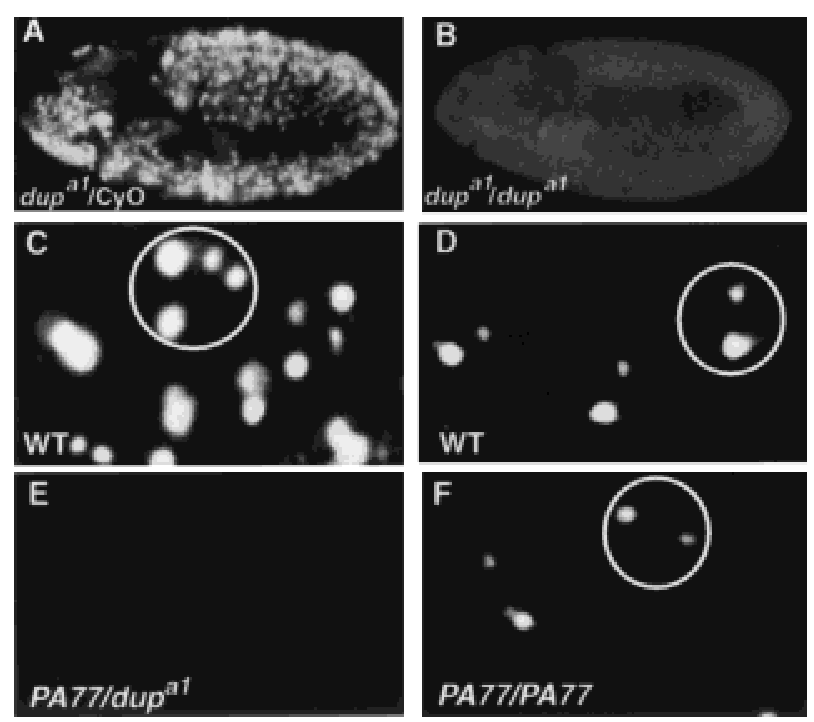

Figure 1. DUP is required for DNA replication in both embryogenesis and oogenesis. $(A, B)$ Embryos pulse labeled with BrdU followed by detection with a fluorescent secondary antibody. (A) A dup ${ }^{a 1} / \mathrm{CyO}$ embryo showing BrdU incorporation in S phase of cycle 16. (B) A dup ${ }^{a 1} /$ dup $^{a 1}$ homozygous mutant embryo that has failed to undergo $S$ phase 16 . The developmental onset of the replication block may not be exactly cycle 16 in every cell, as due to the complex division pattern during these stages we cannot be certain that $\mathrm{S}$ phase of cycle 15 occurred in all of the cells. $(C-F)$ BrdU incorporation in ovarian follicle cells. (C) Labeling of follicle cells from a wild-type stage 10B eggchamber. BrdU incorporation can be seen at the prominent third and $\mathrm{X}$ chromosome chorion regions, and there are two fainter foci (Calvi et al. 1998). (D) In stage 11 wild-type follicle cells BrdU incorporation is only visible at the two chorion regions, with the third chromosome chorion focus being larger than the $X$ chromosome focus (Calvi et al. 1998). (E) Follicle cells from a stage 10B eggchamber from $f_{S}(2) P A 77 /$ dup $^{a 1}$ mutant females. BrdU incorporation is undetectable. $(F)$ Stage 11 follicle cells from a $P A 77 / P A 77$ female. Although BrdU foci are detectable, they are smaller than wild-type controls.

served amplification in only a few stage 10B eggchambers (data not shown). Although incorporated BrdU was detectable in stage 11 eggchambers, the size of the foci was smaller (Fig. 1F), and this is indicative of reduced levels of amplification (Calvi et al. 1998; Royzman et al. 1999). In addition to the amplification defects, we found that genomic replication persisted longer than normal in the $f_{S}(2) P A 77$ homozygous mutant females, because genomic replication was still seen in some follicle cells in late stage $10 \mathrm{~A}$ eggchambers (data not shown).

Occasionally we recovered $f_{S}(2) P A 77 /$ dup $^{a 1}$ female flies. We examined BrdU incorporation in ovaries from these flies and observed that incorporation into the follicle cell genome was reduced relative to wild type. This reduction was most apparent in stage 9 (data not shown). Moreover, we did not detect BrdU incorporation at the chorion loci at any stage (Fig. 1E). These results indicate that genomic replication is decreased in dup mutants and that the initiation of amplification requires functional dup. Thus, dup is essential for replication in mul- tiple stages of development and is required for genomic replication in both mitotic and endo cycles and also for amplification.

\section{Double parked reveals a new family of replication proteins}

To clone the dup gene and identify its protein product we did plasmid rescue using a $\mathrm{P}$ element insert, 1(2) k03308, that failed to complement the lethality of $\operatorname{dup}^{a 1-a 4}$ (see Materials and Methods). The open reading frame is predicted to encode a 743 amino acid, $83.5 \mathrm{kD}$ protein. To confirm that this encoded the DUP protein, we sequenced two of the embryonic lethal alleles, dup ${ }^{a 1}$ and $d u p^{a 3}$, as well as the female-sterile allele, $f_{S}(2) P A 77$ (Fig. 2A). The first two mutations cause stop codons, and the female-sterile allele results in an amino acid substitution. This is consistent with the fact that the dup ${ }^{a 1}$ and $d u p^{a 3}$ mutants have strong phenotypes and are embryonic lethal.

Blast searches with the DUP sequence revealed that it is a member of a family of proteins (Fig. 2B). The carboxy-terminal end of DUP shares very high similarity to a fully sequenced human cDNA the function of which has not been reported (Yu and Gibbs 1997). The human cDNA is $34 \%$ identical and has $52 \%$ conservative changes compared to the carboxy-terminal 446 amino acids of DUP. The human cDNA sequence has been reported to be identical to a sequence $3^{\prime}$ to the human adenine phosphoribosal transferase gene, making it likely that human DUP maps to this region on chromosome 16 (Boyadjiev et al. 1996). DUP also shows high homology to several partially sequenced mouse ESTs. One of these shows $42 \%$ identity and $63 \%$ positives when compared to the carboxy-terminal end of DUP. There are predicted protein orthologs to DUP protein in Arabidopsis thaliana and Caenorhabditis elegans $22 \%$ identity and $36 \%$ positives and $23 \%$ identity and $39 \%$ positives, respectively). Interestingly, DUP is also related to S. pombe Cdt1 (20\% identity and $37 \%$ positives), a protein known to be required for replication initiation (Hofmann and Beach 1994). Alignments of DUP, Cdt1, and the human, C. elegans and A. thaliana orthologs show that these proteins share several conserved motifs, making it likely that they represent members of a family of proteins (Fig. 2B). Arginine 342, which is changed to a cysteine in the $f_{S}(2) P A 77$ allele is conserved in humans, mouse, and $A$. thaliana, consistent with it playing an essential role in the function of DUP. The amino acid sequence of DUP does not reveal predicted biochemical activities.

\section{Double parked localizes to replication origins}

Given the essential role that DUP plays in DNA replication, we wanted to examine the expression and localization of DUP. We generated antibodies to the DUP protein (see Materials and Methods). In stage 2-6 ovarian follicle cells that are proliferating mitotically, some nu- 
Whittaker et al.

Figure 2. DUP defines a new family of replication proteins and shows homology to the $S$. pombe protein Cdt1. (A) DUP is predicted to encode a 743 amino acid, $83.5 \mathrm{kD}$ protein. The $\mathrm{P}$ element (triangle) is inserted 8 base pairs upstream of the beginning of the LD12389 cDNA. DUP contains two introns, one after amino acid 27 that is 96 base pairs long, and one in amino acid 249 that is 1134 base pairs long (inverted Vs). The positions of the mutations in dup ${ }^{a 1}, d_{u p}^{a 3}$, and $d u p^{f_{s(2) P A 77}}$ are indicated by arrows and change amino acids 171, 592, and 342 , respectively. There are two predicted coiled coils, one from amino acids 191-224, and one from amino acids 528-597 (hatched boxes). The two predicted PEST sites are indicated by black boxes and are from amino acids 483-507 and 582-597. (B) The DUP/Cdt1 family. (DM) Drosophila melanogaster; (HS) Homo sapiens; (SP) S. pombe Cdt1; (CE) C. elegans; (AT) A. thaliana. Identical amino acids are boxed and conserved amino acids are shaded.

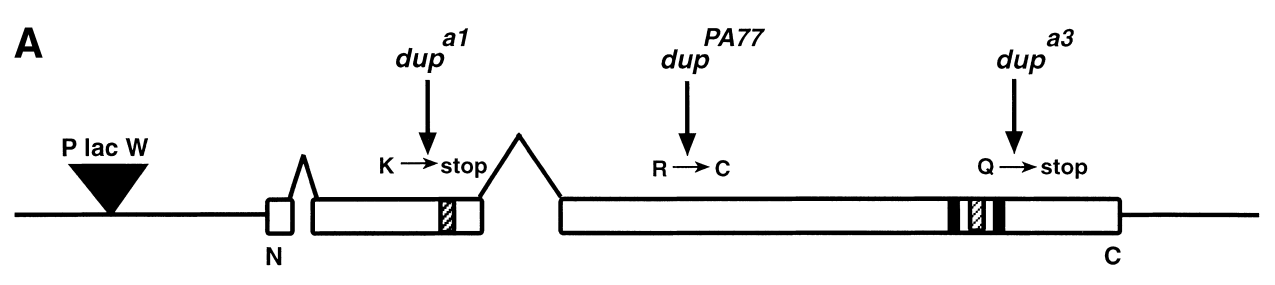

B

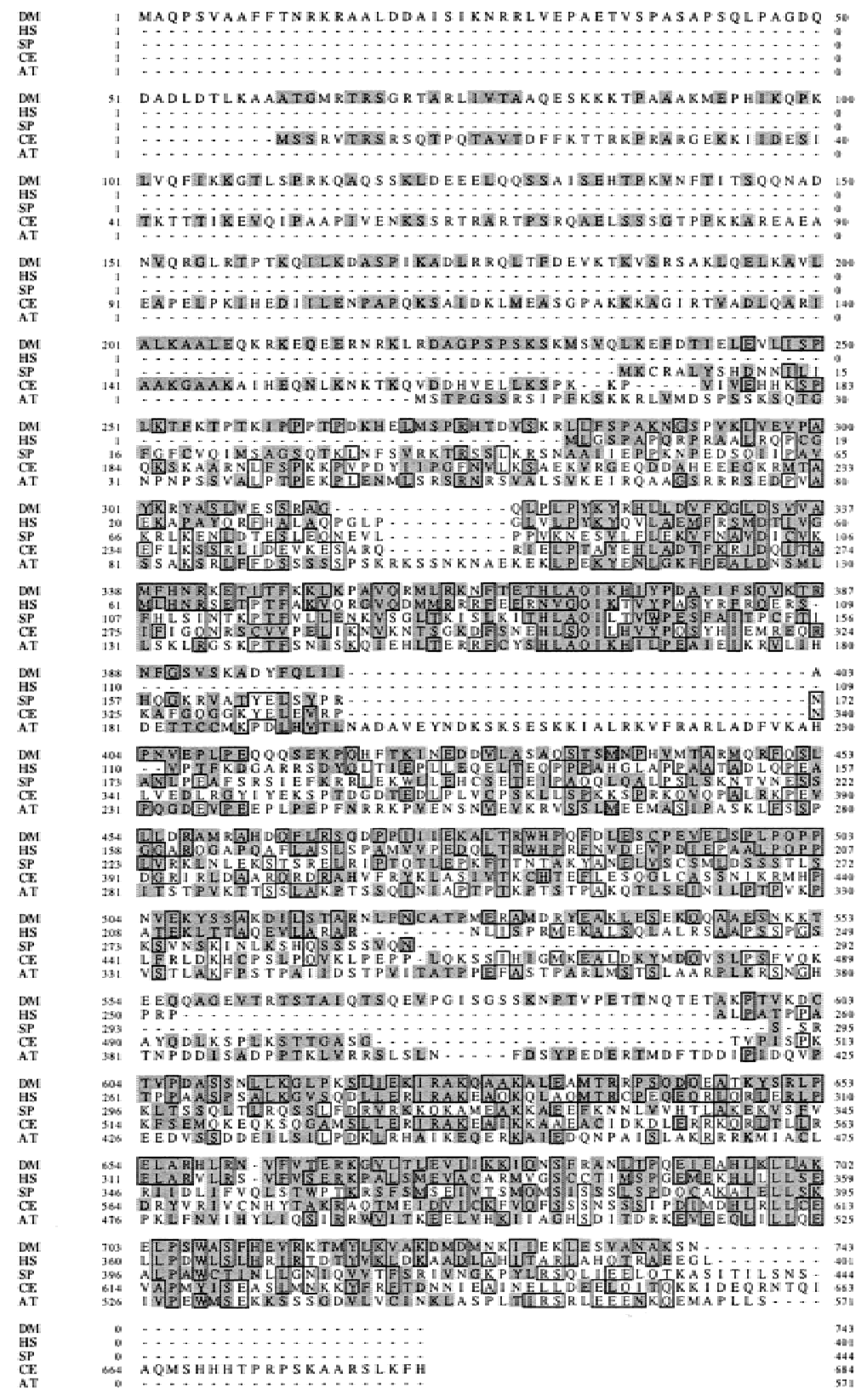

clei stained brightly with anti-DUP antibodies, whereas others were faint (Fig. 3A). This pattern is similar to the pattern of staining observed with MCM and cyclin E antibodies (Calvi et al. 1998; Royzman et al. 1999). It is likely that the cells that stain brightly for DUP, MCM, and Cyclin E proteins are those in S phase. When follicle cells were undergoing endo cell cycles in stages 7-9, DUP appeared to be mainly cytoplasmic, although there was some DUP staining in the nucleus (data not shown). At stage 10A DUP was localized diffusely in the nucleus, but was mostly cytoplasmic (Fig. 3B). At early stage 10B, the stage when amplification was detectable by BrdU incorporation, two subnuclear foci were seen (Fig. 3D), but by late stage 10B primarily one prominent focus was seen in most nuclei (Fig. 3F). A similar localization pattern was seen at stage 11 (data not shown). 

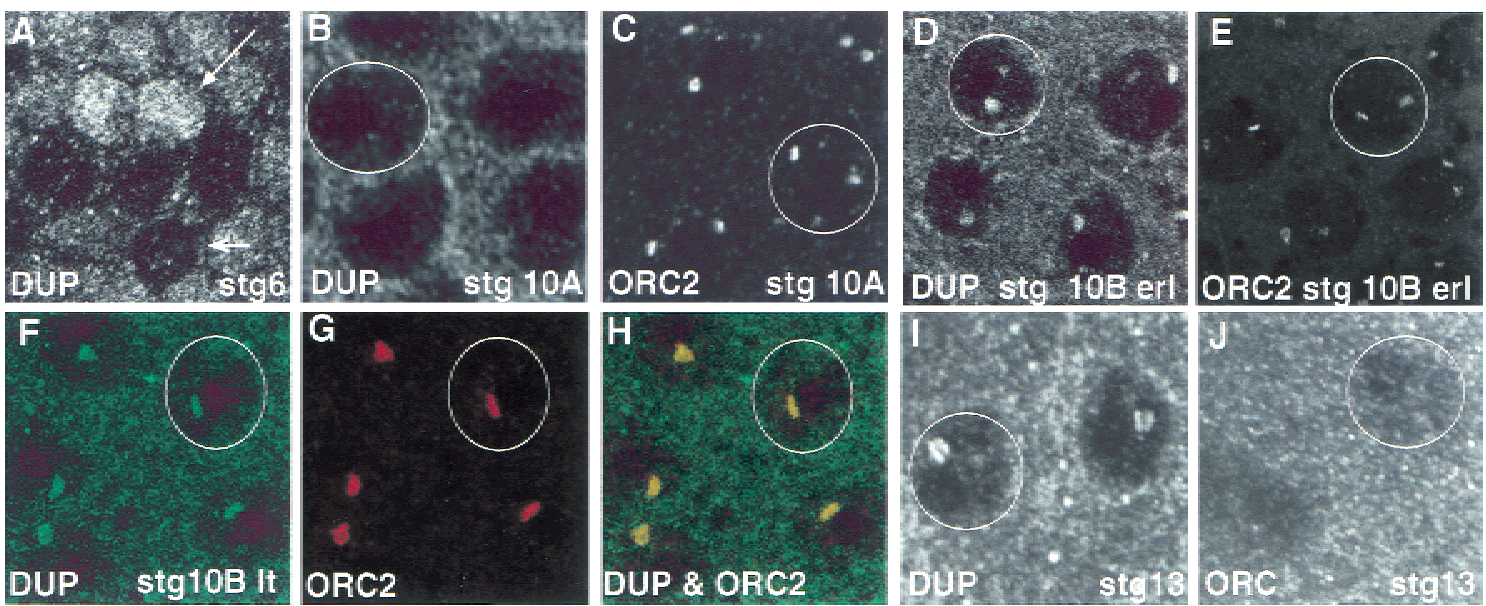

Figure 3. double parked and ORC2 colocalize to amplifying chorion loci. Wild-type eggchambers are shown that have been labeled with ORC2 and/or DUP antibodies. The circles show single follicle cell nuclei. (A) A stage 6 eggchamber stained with DUP antibodies. Some follicle cell nuclei stain brightly (large arrow), whereas others have low levels of DUP (small arrow). (B) A stage 10A eggchamber stained for DUP, showing low nuclear levels of the protein at this stage. $(C)$ In stage 10A eggchambers stained with ORC2 antibodies two prominent foci of staining are visible. $(D)$ By early stage 10B DUP protein is present at two foci, the same pattern as for ORC localization $(E) \cdot(F-G)$ Wild-type stage 10B late eggchambers double labeled for DUP and ORC. $(F)$ DUP staining is in green. There is a single focus of nuclear localization. $(G)$ ORC2 is also present as a single focus. $(H)$ Merged image shows that DUP and ORC2 colocalize. (I) DUP staining in a stage 13 eggchamber. (J) A stage 13 eggchamber stained for ORC2 which is no longer detectable.

The subnuclear localization pattern of DUP at stages $10 \mathrm{~B}$ and 11 is strikingly similar to that seen for ORC2 and ORC1, which have been shown to localize to the sites of chorion gene amplification at these stages (Asano and Wharton 1999; Royzman et al. 1999). To determine if ORC2 and DUP colocalize, ovaries were stained simultaneously with ORC2 and DUP antibodies. At stage 10B DUP and ORC2 colocalized in the follicle cells, although the DUP staining appeared more diffuse than that of ORC2 (Fig. 3F-H). By stage 11 only one focus of ORC2 was seen in follicle cell nuclei. DUP remained detectable at two foci in most nuclei, but one focus was clearly brighter than the other (data not shown).

Two interesting differences were seen in the localization pattern of ORC2 and DUP. First, at stage 10A, ORC2 had already localized to subnuclear foci. DUP, in contrast, was still diffusely localized in the nucleus (Fig. 3 , cf. B and C). Secondly, ORC2 was not seen to localize to origins after stage 11 , but BrdU incorporation continues for another seven hours, until stage 13 (Fig. 3J; Royzman et al. 1999). Prior results suggested that this BrdU incorporation is due to elongation (Royzman et al. 1999). The levels of localized ORC1 protein also diminish after stage 10B (data not shown). Interestingly, DUP was undiminished and remained detectable at subnuclear foci until stage 13, indicating that it may function during elongation (Fig. 3I). Alternatively, DUP could have no role in elongation but is not cleared from chromatin until after elongation has been completed.

Because ORC is localized specifically before DUP, we tested whether ORC function was a prerequisite for DUP localization at replication foci. There is a female-sterile mutation in the orc2 gene, $f_{S}(2) 293$, that is defective in amplification (Landis et al. 1997). We first examined the effect of the orc2 mutation on ORC localization. In the
$f_{S}(2) 293$ mutants, the amount of ORC2 was greatly reduced or undetectable at specific foci during amplification (data not shown). We found that DUP was not localized to specific foci in this mutant in many follicle cell nuclei (Fig. 4B). In other follicle cells, DUP could be seen at one focus, but the levels were greatly reduced
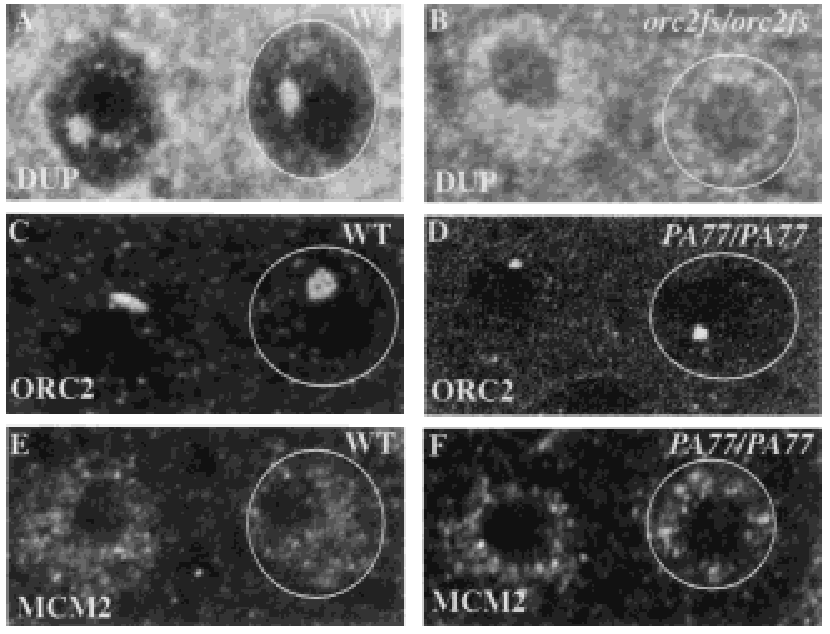

Figure 4. Requirements for ORC and DUP localization. (A) Wild-type (WT) stage 10B eggchambers stained with DUP antibodies show a prominent focus of localization. $(B)$ This localization is reduced in orc2 mutants, whereas the overall levels of protein in some of the nuclei are higher. $(C)$ Wild-type stage 10B eggchamber stained with anti-ORC2. (D) In the dup fs(2)PA77 mutants ORC2 localizes in the normal ring structure (Austin et al. 1999), but its size is reduced. (E) A stage 10B eggchamber stained with antibodies against MCM2. The protein does not localize specifically to amplifying foci. $(F)$ There is no detectable alteration or reduction of MCM2 protein in the dup fs $(2) P A 77$ mutants. 
(data not shown). This suggests that ORC2 is required for DUP localization. Interestingly, in some nuclei, high nuclear levels of DUP were observed (Fig. 4B). The levels were higher than those seen during stages 7-10A. Additionally, DAPI staining of the nuclei didn't indicate that morphology of the cells was altered. This may suggest that ORC2 is required either indirectly through its requirement for replication, or directly, to prevent nuclear accumulation of DUP. It may be that DUP enters the nucleus independently of ORC, but that ORC is required to localize it to the amplifying regions.

To determine if DUP was necessary for continued ORC localization, we examined ORC2 localization in the $f_{S}(2) P A 77$ mutant. The shape and number of ORC2 foci was normal in the mutant follicle cells, but the size was smaller (Fig. 4D). One interpretation of these results is that DUP plays a role in loading or maintaining ORC2 at the amplification origins. Because the shape and number of foci seen was appropriate for the stages examined, we think it more likely that the decrease in levels of ORC2 localization results from lower levels of DNA replication, causing decreased copy number of the origins.

To further delineate the time at which DUP acts during replication, we wanted to determine if dup is required for normal levels of the MCM proteins in the nuclei. MCMs proteins have been shown to be present uniformly in the nuclei of stages 10B-13 follicle cells, and they do not localize specifically to the amplifying regions as do the ORC and DUP proteins (Royzman et al. 1999). In ovaries from $f_{S}(2) P A 77$ homozygous mutant females incubated with antibodies to MCM2 no alterations in the levels was detected (Fig. 4, cf. E and F).
DUP is cell cycle regulated and is a downstream target of the E2F transcription factor

Given the critical role DUP plays in replication we postulated that like other replication genes such as cyclin $E$ and PCNA dup is transcriptionally regulated by the E2F transcription factor (Duronio et al. 1995). To examine the expression pattern of dup transcript, embryos of various developmental stages were hybridized in situ with dup riboprobes. The pattern of expression of dup transcript was very similar to that of other $S$ phase genes (Duronio and O'Farrell 1994). High levels of maternal transcript were present during cycles 1-13 (Fig. 5A). At cycle 14, the transcript was downregulated (data not shown). Ubiquitous expression of dup transcript was seen during the postblastoderm divisions (Fig. 5B). After cycle 16 , concurrent with the addition of a G1 phase, dup transcript was downregulated in all tissues with the exception of the proliferating central nervous system (CNS) and peripheral nervous system (PNS). At cycle 17, dup transcript was expressed just prior to $S$ phase and down regulated after $S$ phase in the endoreplicating gut (Fig. 5C). Thus, like other $S$ phase genes, expression of dup is regulated at the G1-S transition of the cell cycle.

To test if the cell cycle transcription of dup is dependent on E2F, embryos homozygous for a null allele of the dE2F1 subunit, $d E 2 F^{91}$ were collected and hybridized with dup riboprobes. The levels of dup transcript were decreased in the endoreplicating gut and appeared to be slightly decreased in the CNS (Fig. 5D). A similar effect on dup transcript was seen in embryos that were homozygous mutant for the other subunit of the E2F transcription factor, of the genotype $d D P^{a 2}$ (Fig. 5E). Thus, dup is a downstream target of the E2F transcription factor. In-
Figure 5. dup is expressed in a pattern similar to $\mathrm{S}$ phase genes and is regulated by E2F and cyclin E. Embryos were hybridized in situ with riboprobes to the dup cDNA. (A) A wild-type stage 3 embryo, showing high levels of maternal dup transcript. (B) A wild-type stage 7 embryo showing that dup is ubiquitously expressed during the postblastoderm divisions. Although higher levels of expression were seen in the gut primordia and neural tissues, these also stained darker in control embyros hybridized with the sense probe. Therefore, these tissues have a higher intrinsic background signal, possibly due to a higher cell density. (C) A stage 12 wild-type embryo reveals that dup is downregulated after cycle 16 and expressed in at the G1-S transition in endo cycling cells. (amg) Anterior midgut; (cmg) central midgut; (pmg) posterior midgut; (hg) hind gut; (mt) malpighian tubules. (D) A homozygous $d E 2 F 1^{91}$ / $d E 2 F 1^{91}$ mutant embryo with decreased levels of dup transcript. (E) A homozygous $d D P^{a 2} / d D P^{a 2}$ mutant embryo, and $(F)$ a homozygous cyclinE $E^{1(2) 305} /$ cyclinE $E^{1(2) 305}$ mutant embryo with persisting expression of dup transcript in the central midgut.
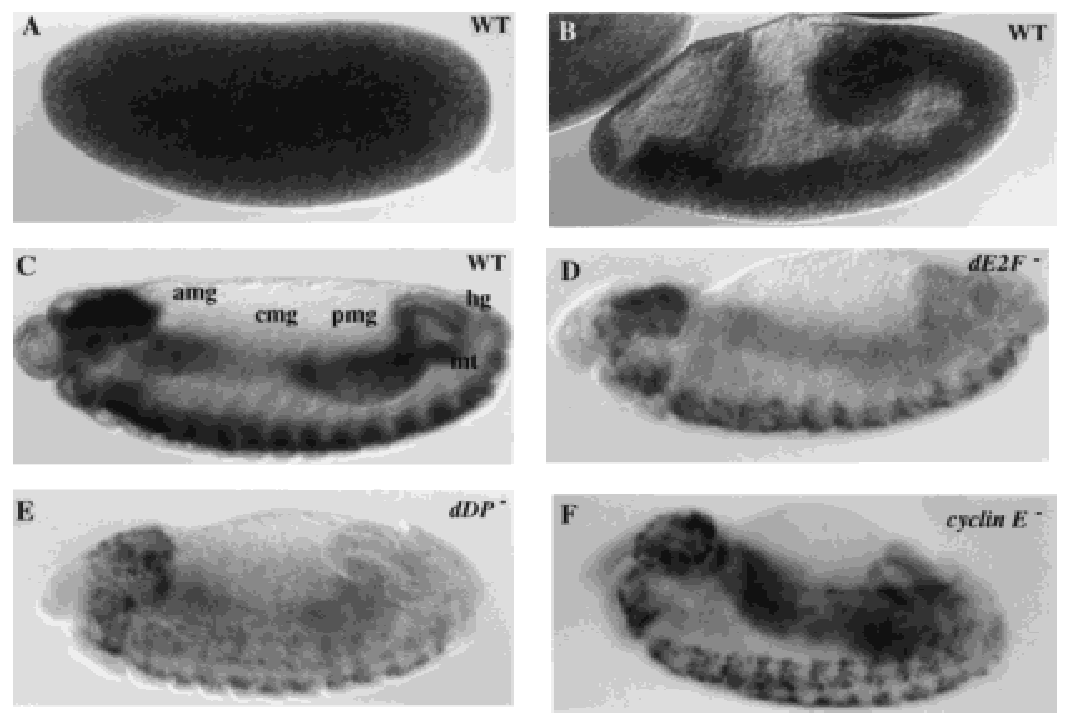
terestingly, cdt1 transcription is also cell-cycle regulated. Expression of $c d t 1$ is controlled by the G1-S transcription factor Cdc10 that, like E2F, regulates transcription of many genes required for $\mathrm{S}$ phase (Hofmann and Beach 1994). This suggests that cell cycle control of dup may be conserved, and DUP may prove to be an important downstream target of E2F in mammalian cells.

Cyclin $\mathrm{E}$ is required to regulate positively the transcription of $S$ phase genes in the nervous system and to downregulate these transcripts in endo cycling cells (Duronio and O'Farrell 1995; Sauer et al. 1995). We found that the cyclinE $E^{1(2) 305}$ and cyclin $E^{P Z 5}$ mutations and a cyclin $E$ deficiency, $D f(2 L) T E 35 D 1$, have similar effects on dup transcripts. In these embryos, dup was not downregulated properly in the endoreplicating gut such that dup transcripts persisted at higher levels than wild type in the anterior midgut, central midgut, and posterior midgut in later embryonic stages (Fig. 5F; data not shown). In cyclin $E$ mutant embryos dup transcripts were reduced in the CNS, although not to as great an extent as other $\mathrm{S}$ phase genes. Thus, dup expression also is regulated by cyclin $\mathrm{E}$.

In dup mutants, cell cycle events normally dependent on $S$ phase are regulated improperly

If $S$ phase is blocked during the postblastoderm divisions by treatment with aphidocolin, the cell cycle arrests and mitosis does not occur (Foe et al. 1993). In yeast it has been demonstrated as well that arrest in $S$ phase stops the cycle and blocks mitosis (for review, see Li and Deshaies 1993). In contrast, in yeast mutation of the Cdc18/Cdc6 protein required to initiate DNA replication does not block mitosis even though DNA replication does not take place (Kelly et al. 1993; Piatti et al. 1995). Therefore, we wanted to test whether mitosis occurred after the S phase block in dup mutant embryos to determine whether this checkpoint was functional in dup mutants.

We used the phosphoH3 epitope (PH3) present on condensed chromosomes to test for the presence of mitotic cells in dup mutant embryos (Hendzel et al. 1997). Normally, in embryos that have completed cycle 16, PH3 antibodies label mitotic cells solely in the CNS and the PNS (Fig. 6E). No labeling is seen in the G0 epidermal cells or in the endo cycling cells. In contrast, in dup $p^{a 1} /$ $d_{u p}{ }^{a 1}$, dup ${ }^{a 1} / d_{u p}{ }^{a 3}$, and dup ${ }^{a 3} / d_{u p} p^{a 3}$ mutant embryos many cells in the epidermis labeled with anti-phospho H3 antibodies (Fig. 6F; data not shown). There were also some labeled cells in the gut in addition to the CNS and PNS. This defect was first manifested at mitosis 16, after the block in S phase was observed (Fig. 6, cf. A and B). The mitotic block occurs after the failure of DNA replication because closer examination of the chromosomes showed that they were $2 \mathrm{~N}$ (Fig. 6D). In addition, the number of nuclei in stage 11 embryos indicates that mitosis 15 takes place in dup mutant embryos. The number of nuclei within each segment of the mutant embryos is about half that of wild type, implying failure of the last division of embryogenesis (mitosis 16) but not the last two divisions (data not shown). Although we cannot exclude the possibility that in some cells mitosis 15 does not occur, it does take place in the majority of the cells.

The labeled cells were blocked in an abnormal mitosis 16 with unreplicated chromosomes (Fig. 6, cf. C and D). The chromosomes appeared over-condensed and in some cells fragmented. No telophase figures were detected, and thus the mutant cells do not complete mitosis. After stage 12 , as development progressed, the number of antiphospho H3 labeled cells decreased. Because we saw no indication that these cells completed mitosis, this may be due to cell death. However, it is also possible that these cells aborted mitosis and returned to G0 or G1
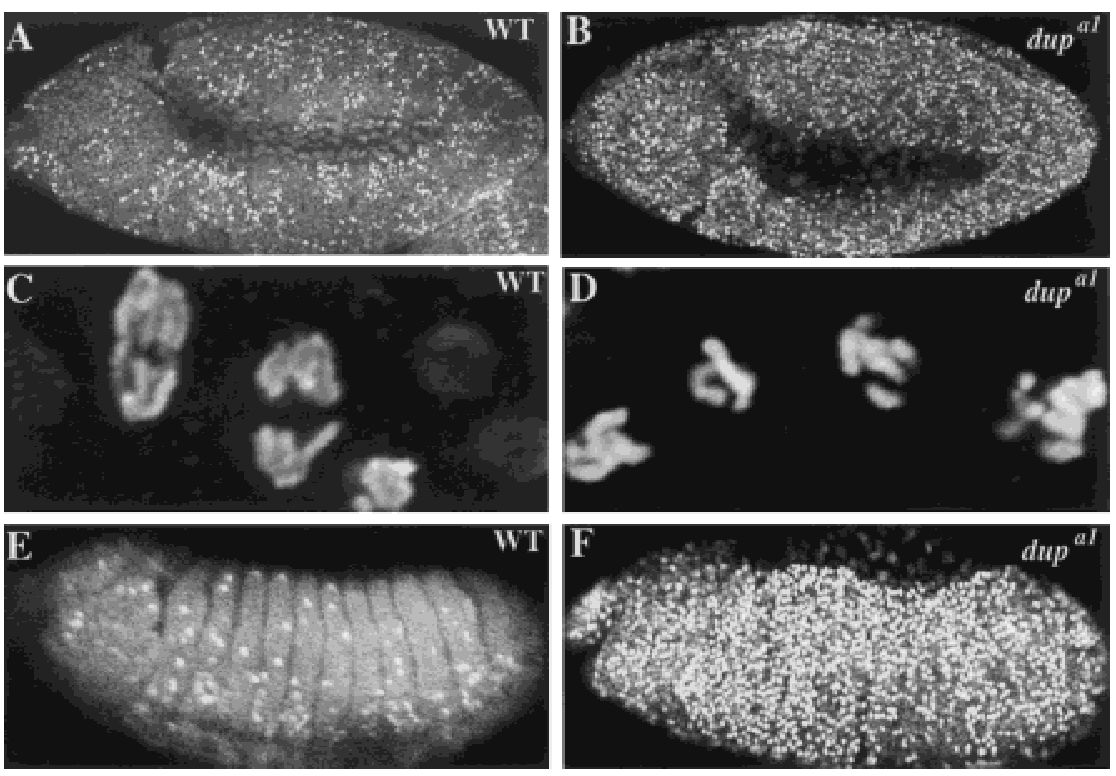

Figure 6. Despite a failure to complete S phase, embryos homozygous for dup mutations progress into mitosis. All embryos were labeled with antibodies to phosphorylated histone H3. $(A, C, E)$ Wild-type embryos; $(B, D, F)$ dup ${ }^{a 1} /$ dup $^{a 1}$. (A) Wild-type stage 10 embryo, notice PH3 labeling in discrete domains in the epidermis. $(B)$ In this dup mutant stage 10 embryo $\mathrm{PH} 3$ is present throughout the epidermis. $(C)$ Enlargement of $A$. $(D)$ Enlargement of $B$. (E) Wild-type stage 12 embryo, with $\mathrm{PH} 3$ only detectable in the mitotically proliferating central nervous system and peripheral nervous system. (F) Stage 12 dup $^{-}$mutant embryo in which $\mathrm{PH} 3$ is still detectable in the epidermis. 
phase. Thus, despite the failure to undergo $S$ phase, cells enter mitosis in dup mutant embryos.

The dup mutant embryos are defective in another cell cycle process in that they fail to downregulate transcripts that were induced at the G1-S transition. This phenotype was the basis by which mutations in dup were originally isolated in a screen designed to recover mutations in G1-S transcription. PCNA transcripts remain at high levels after $\mathrm{S}$ phase 16 in dup mutants, rather than being downregulated. We wished to determine if dup mutations affect other $\mathrm{S}$ phase genes similarly. To test this, in situ hybridization with cyclin $E$ and RNR2 riboprobes was done on dup mutant embryos. In all four alleles of dup, $S$ phase transcripts failed to be down regulated in both the epidermis and the endo cycling cells (Fig. 7C,D). In older stage 14 embryos the level of transcript appeared to decrease (data not shown). Similar to staining with anti-phospho $\mathrm{H} 3$, this could be due to cell death occurring in the epidermis or cells reentering G0 or G1 phase. This failure to downregulate S phase transcripts is not the result of mitotic arrest in dup mutants, because pimples mutant embryos arrested at metaphase of cycle 16 downregulate transcripts properly (Royzman 1998). This transcriptional phenotype may indicate that dup plays a direct role in transcriptional regulation. Alternatively, the failure to down regulate $S$ phase transcripts may simply result from a failure to complete S phase. We favor the later interpretation. First, the replication phenotype precedes that of the transcription phenotype. Also, the female-sterile mutation in dup does not affect the levels of PCNA transcripts in the ovary (data not shown).

\section{Discussion}

We have isolated a new gene, double parked, in Drosophila that is essential for replication in multiple stages of development. DUP is a member of a conserved family of replication proteins with homologs in organisms ranging from $S$. pombe to humans. DUP colocalizes with ORC2 to chorion amplification foci, suggesting that it is part of the replication machinery. The dup mutants are striking also because they lack the checkpoint that would normally prevent mitosis if replication were incomplete. This may be either because DUP is part of this regulatory pathway or because $S$ phase does not proceed sufficiently far in the mutants for the cells to sense that replication is incomplete. Furthermore, dup mutants fail to downregulate $S$ phase transcripts on the addition of the first G1 phase in embryogenesis, suggesting that completion of $S$ or passage through a certain point in $S$ phase may be required for this downregulation.

Our results show that DUP is essential for replication at multiple stages of development, during the post blastoderm mitotic cycles, the endocycles, and amplification that occurs during oogenesis. DUP is probably required for $S$ phase in the early embryonic cycles as well, as the maternal pools of protein could account for why a replication defect is not detected until cycle 16. The mutant phenotypes do not distinguish whether DUP acts in initiation of DNA replication, elongation of replication forks, or both. Two results suggest that DUP acts after ORC in replication. First, ORC2 is still localized in dup mutants. Second, DUP localization to foci does not become apparent until after ORC2 is localized. The fact that no BrdU incorporation is detectable in strong dup mutants suggests an early block in replication and a role for DUP in initiation. Because DUP foci persist longer than ORC2 foci and DUP remains localized during stages when amplification elongation rather than initiation is occurring, DUP may function in elongation as well. This does not exclude a role of DUP in initiation, as the MCM proteins are part of the preinitiation complex at origins but also appear to move with the replication fork (Aparicio et al. 1997). Further experiments will be required to determine whether DUP is present both at moving replication forks as well as the amplification origins.

The similarity between DUP and the $S$. pombe protein Cdt1 suggests a model for how DUP functions in replication. Mutations in $c d t 1$ block DNA replication initiation (Hofmann and Beach 1994). Recent experiments show that Cdt1 accumulates in the nucleus in G1 and is a component of the prereplicative complex (Nishitani et al. 2000). Cdt1 protein is in a complex with the Cdc18

\section{cyclin $E$}

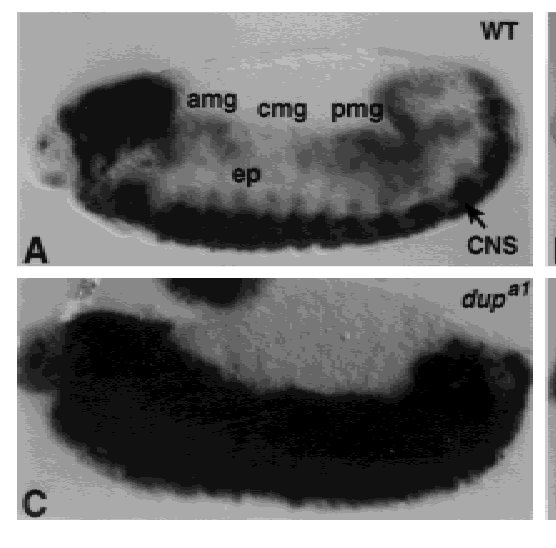

\section{RNR2}

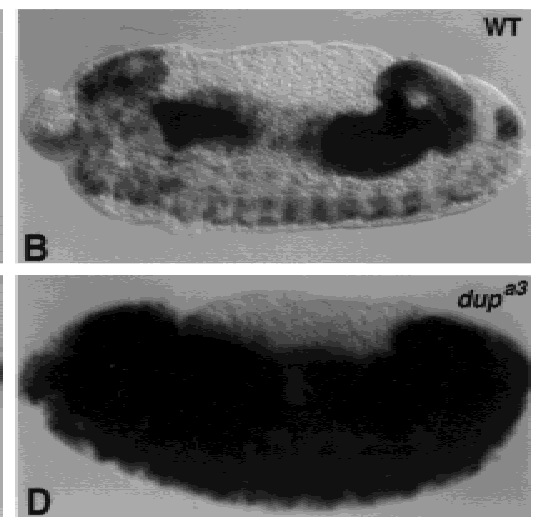

Figure 7. S phase transcripts fail to switch from ubiquitous to patterned expression in dup homozygous mutant embryos. Wild-type and dup homozygous mutant embryos were hybridized in situ with cyclin $E(A, C)$ and RNR2 $(B, D)$ riboprobes. $(A, B)$ Wild-type embryo; $(C)$ dup $p^{a 1} /$ dup $^{a 1}$ stage 13 embryo; $(D)$ dup $^{a 3} /$ dup $^{a 3}$ homozygous mutant embryo. Note the high levels of expression of $\mathrm{S}$ phase genes in the epidermis of the embryos shown in $C$ and $D$. (amg) Anterior midgut; (cmg) central midgut; (pmg) posterior midgut; (ep) epidermis; (CNS) central nervous system. 
protein, the $S$. pombe homolog of the Cdc6 initiator protein. In addition, loading of MCM proteins onto chromatin is blocked in cdt1 mutants (Nishitani et al. 2000). An ortholog to Cdt1 was identified recently in Xenopus and shown to be required for DNA replication in vitro to load MCM proteins (Maiorano et al. 2000). Consistent with the results on DUP localization, Xenopus Cdt1 association with chromatin is dependent on ORC. However, in Xenopus extracts Cdt1 dissociates from chromatin after the initiation of DNA replication (Maiorano et al. 2000). By analogy to Cdt1, DUP may be needed in conjunction with Cdc6/Cdc18 to help load MCM proteins onto the origins, but also to maintain them at the replication fork. Because the MCM proteins are not specifically localized during amplification in the follicle cells, we could not detect an effect of the dup mutations on MCM localization (Royzman et al. 1999). Therefore, test of this model will require biochemical approaches.

Using the sequence of DUP we identified homologs in human, Mus musculus, A. thaliana, and C. elegans. This together with the identification of a Xenopus ortholog establishes that Cdt1 and DUP are members of a family of replication proteins. The extent of conservation between the Drosophila DUP protein and those predicted from the human and mouse cDNAs makes it likely that DUP will be critical for replication in mammalian cells. Interestingly, no DUP homolog has been found in S. cerevisiae. DUP may be needed for replication initiation in eukaryotes that have more complex origins of replication, and hence not present in S. cerevisiae. As sites of initiation in these eukaryotes are less specific than in $S$. cerevisiae, it is possible that DUP plays a role in helping to direct MCMs to the appropriate origins. This would be especially useful in higher eukaryotes where origin usage varies by cell type and time in development. Alternatively, S. cerevisiae may contain an analogous protein that is sufficiently diverged so as to have not been recognized in similarity searches. The MCM10 protein is needed to load MCM proteins into the $S$. cerevisiae prereplicative complex, so MCM10 could do the same function as Cdt1/DUP in metazoans (Homesley et al. 2000).

The dup mutations uncouple the dependency of mitosis on completion of S phase. This may be because dup is part of the machinery that implements this checkpoint. However, precedents in yeast support the hypothesis that the failure to engage this checkpoint in dup mutants is a consequence of replication not initiating and not producing stalled intermediates that can be recognized (Kelly et al. 1993; Li and Deshaies 1993; Piatti et al. 1995). In both $S$. cerevisiae and $S$. pombe mutations that block initiation of DNA replication do not block progression into mitosis, whereas arrest during elongation stages of DNA replication does. Therefore, if DUP is indeed critical for initiation of DNA replication, the absence of DNA synthesis may account for the checkpoint failure without any direct involvement of DUP in the machinery that signals the checkpoint.

The mutations in dup also reveal a likely requirement for the initiation of $S$ phase for the subsequent downregulation of $S$ phase transcripts. Interestingly, muta- tions in string that block in G2 phase and prevent $S$ phases 15 and 16 do not prevent downregulation of transcripts (Duronio and O'Farrell 1994), nor does arrest in metaphase by mutations in three rows or pimples (Royzman 1998). Therefore, the failure to downregulate the level of S phase transcripts in dup mutants is not the consequence of a simple block in the cell cycle or complete prevention of $S$ phase. It is logical that a cell would not downregulate the transcripts encoding products required for DNA replication until initiation had occurred, possibly reflecting a strategy to drive S phase to completion by maintaining high levels of the replication machinery. Interestingly, in S. pombe cdc18 transcript is not downregulated if $S$ phase is blocked with hydroxyurea, indicating that downregulation may require the completion, not merely the initiation of DNA replication (Baum et al. 1997).

The identification of dup by its mutant phenotype highlights the power of Drosophila genetics for providing insights into the regulation of DNA replication that extend into mammalian systems. In addition these mutations shed light on the coordination of $S$ phase with subsequent cell cycle steps such as the downregulation of $S$ phase genes and the onset of mitosis. The defined replicon present in amplifying follicle cells to which DUP localizes will provide the necessary resolution to delineate the molecular mechanism by which DUP controls replication in metazoans.

\section{Materials and methods}

Strains

The dup ${ }^{a 1-a 4}$ mutations were isolated in a screen that has been previously described (Royzman et al. 1997). All four alleles fail to complement the lethality of the 1(2)51Ec mutation (Underwood et al. 1990). The $f_{s}(2) P A 77$ line was provided by Trudi Schupbach (Schupbach and Wieschaus 1989). 1(2)k03308 was provided by Todd Laverty and has been described elsewhere (Torok et al. 1993; Roch et al. 1998). The deficiency Df(2R)JP1 was provided by the Bloomington Drosophila stock center. The Ubx-lacZ CyO balancer used to identify homozygous embryos has been described (McCall et al. 1994). The lethal phase of the dup ${ }^{a 1-a 4}$ alleles over $D f(2 R) J P 1$ was determined as described previously (Royzman et al. 1997).

Cloning of dup

The dup gene was cloned by identifying a $\mathrm{P}$ element insertion in the gene and recovering flanking DNA. To show that $1(2) \mathrm{k} 03308$ disrupts the dup gene 72 independent excision lines were generated by crossing in the transposase gene and screening for loss of the $\mathrm{w}^{+}$eye color marker on the P element. Twenty-five of these excision lines complemented the lethality of the dup ${ }^{a 1}$ or $d u p^{1(2) 51 E c}$ lines, indicating they are precise excisions and demonstrating lethality can be reverted by removal of the P element. Genomic DNA 3' to the P lacW element was isolated by plasmid rescue. In situ hybridization was performed on polytene chromosomes using a biotinylated probe made from the plasmid containing the rescued genomic DNA, as described (Pardue 1994). This probe hybridized to the region 51F11-12 where 1(2)k03308 has been reported to map (Spradling et al. 1995). The flanking genomic probe was sequenced by Research Genetics. 
Using the Berkeley Drosophila Genome Project's blast server this sequence was found to be homologous to a clot of ESTs, 845 (http://www.fruitfly.org/; D. Harvey, L. Hong, M. Evans-Holm, J. Pendleton, C. Su, P. Brokstein, S. Lewis, and G.M. Rubin, BDGP/HHMI Drosophila EST Project, unpubl.). The most 5' cDNA, LD12389, and the genomic DNA were sequenced by Research Genetics. The ORF finder program and Edit seq programs were used to predict the DUP ORF. The predicted start site of this ORF fits the qualifications of a typical Drosophila start site well (Cavener 1987). The GenBank accession number for the cDNA and protein sequences is AF279146. The predicted coiled coils were found using the pair coil and multi coil programs (Berger et al. 1995; Wolf et al. 1997). The predicted PEST sites were found using the PEST find program (Rechsteiner and Rogers 1996).

To sequence the dup alleles, genomic DNA was isolated from single embryos using a modification of the single fly DNA preparation protocol (Gloor and Engels 1992). To distinguish between homozygous and heterozygous embryos, PCR amplification was done using primers to $\mathrm{lacZ}$ present on the $\mathrm{CyO}$ $U b x-l a c Z$ balancer carried by the heterozygotes. The genomic region encoding DUP was then amplified by PCR from the genomic DNA samples lacking lacZ product, and the product was sequenced directly by Research Genetics. Genomic DNA was isolated from females homozygous for the $f_{S}(2) P A 77$ mutation, PCR amplified, and sequenced. Because $f_{S}(2) P A 77$ was induced in a different strain than the dup lines isolated in our screen, we also sequenced the dup genomic region in a cortex mutant isolated in the same screen as $f_{S}(2) P A 77$ (Schupbach and Wieschaus 1989). The arginine to cysteine change in the $f_{S}(2) P A 77$ line is not present in cortex flies.

The DUP orthologs were isolated using Gapped Blast (Altschul et al. 1997). The alignments were done using the Clustal X program. To allow alignment of the full human sequence with DUP it was necessary to add or subtract 1 base pair in one case and 2 base pairs in another place to remove in frame stops. The accession numbers for the DUP orthologs are as follows: human (AF070552); M. musculus ESTs (AI605978, AA139554, and AA032327); and A. thaliana (AAD20672). The C. elegans ortholog can be found at the following URL, http:// www.sanger.ac.uk/cgi-bin/nph-getblast?wormpub/wormpep current+Y54E10A 156.A.

\section{Antibody preparation}

Antibodies were prepared in guinea pigs against a fusion protein between DUP and GST. Two-thirds of DUP was fused to GST by digesting the LD12389 cDNA with HpaI and SspI and ligating it into the pGEX $3 \mathrm{X}$ vector digested with SmaI. The proteins were expressed in the $\mathrm{DH} 5 \alpha$ strain using a modification of the protocol outlined in Tang et al. (1998). The protein was sent to Covance for antibody production.

The DUP antibodies recognize a prominent band on an immunoblot of wild-type embryo extracts. This band was greatly reduced in extracts made from dup ${ }^{a 1} / d_{u} p^{a 1}$ homozygous mutant embryos (data not shown). The staining pattern observed in follicle cells also was affected by the dup mutations. DUP localization to subnuclear foci was greatly reduced in $f_{S}(2) P A 77$ mutant follicle cells, and it was completely absent in ovaries from $f_{S}(2) P A 77 /$ dup $^{a 1}$ females. To ensure further the specificity of the DUP antibodies, ovaries from wild-type females were incubated with DUP antibodies that had been incubated for one hour with $3 \mu \mathrm{g}$ or $400 \mathrm{ng}$ of GST-DUP, GST-SIC1, or buffer alone. DUP staining was absent in ovaries treated with antibodies incubated with $3 \mu \mathrm{g}$ GST-DUP and greatly reduced in samples treated with $400 \mathrm{ng}$ GST-DUP. It was unaffected in samples treated with antibodies incubated with GST-SIC1 or elution buffer.

\section{Cytology}

BrdU labeling and antibody staining on embryos and ovaries was done as described previously (Royzman et al. 1997, 1999). Heterozygous embryos were distinguished from homozygous embryos by the presence of a balancer chromosome expressing $\beta$ galactosidase in the Ubx pattern. This was detected by the use of a mouse anti- $\beta$ galactosidase antibody used at a concentration of 1:100. Preparation of riboprobes and in situ hybridization to embryos was done as described previously (Tautz and Pfeifle 1989). The anti-ORC2 antibodies were provided by Richard Austin and Steve Bell and used at a dilution of 1:2500. AntiDUP antibodies were used at a dilution of 1:1000. Microscopy for fluorescently labeled embryos and ovaries was done using either a Biorad MRC600 or a Zeiss LSM S10 confocal microscope. Microscopy of hybridized embryos was done on a Zeiss Axiophot microscope.

\section{Acknowledgments}

We are grateful to Hideo Nishitani, Zoi Lygerou, and Paul Nurse for communicating their unpublished observations. We thank Rick Austin and Steve Bell for providing antibodies, helpful discussions, and comments on the manuscript. Ken Moberg and Kim Dej also provided helpful suggestions on the manuscript. The Berkeley Drosophila Genome Project provided the P element insertion strain and EST clones of dup. The microscopy was done in the Keck Imaging Facility at the Whitehead Institute. A.W. and I.R. were supported by a predoctoral training grant from the National Institutes of Health, and this research was supported by grants from the National Institutes of Health to T.O.-W.

The publication costs of this article were defrayed in part by payment of page charges. This article must therefore be hereby marked "advertisement" in accordance with 18 USC section 1734 solely to indicate this fact.

\section{References}

Altschul, S.F., Madden, T.L., Schaffer, A.A., Zhang, J., Zhang, Z., Miller, W., and Lipman, D.J. 1997. Gapped BLAST and PSI-BLAST: A new generation of protein database search programs. Nucleic Acids Res. 25: 3389-3402.

Aparicio, O.M., Weinstein, D.M., and Bell, S.P. 1997. Components and dynamics of DNA replication complexes in S. cerevisiae: Redistribution of MCM proteins and Cdc45p during S phase. Cell 91: 59-69.

Asano, M. and Wharton, R.P. 1999. E2F mediates developmental and cell cycle regulation of ORC1 in Drosophila. EMBO J. 18: $2435-2448$.

Austin, R.J., Orr-Weaver, T.L., and Bell, S.P. 1999. Drosophila ORC specifically binds to $A C E 3$, an origin of DNA replication control element. Genes \& Dev. 13: 2639-2649.

Baum, B., Wuarin, J., and Nurse, P. 1997. Control of S-phase periodic transcription in the fission yeast mitotic cycle. EMBO I. 16: 4676-4688.

Bell, S.P. and Stillman, B. 1992. ATP-dependent recognition of eukaryotic origins of DNA replication by a multiprotein complex. Nature 357: 128-134.

Berger, B., Wilson, D.B., Wolf, E., Tonchev, T., Milla, M., and Kim, P.S. 1995. Predicting coiled coils using pairwise-residue correlations. Proc. Natl. Acad. Sci. 92: 8259-8263. 
Boyadjiev, S.A., Sahota, A., and Tischfield, J.A. 1996. Identification and application of polymorphisms flanking the human adenine phosphoribosyltransferase gene. Hum. Mutat. 8: 214-215.

Calvi, B.R. and Spradling, A.C. 1999. Chorion gene amplification in Drosophila: A model for origins of DNA replication and S phase control. In Genetic approaches to eukaryotic replication and repair (ed. P. Fisher), pp. 407-417. Academic Press, New York, NY.

Calvi, B.R., Lilly, M.A., and Spradlng, A.C. 1998. Cell cycle control of chorion gene amplification. Genes \& Dev. 12: $734-744$.

Cavener, D. 1987. Comparison of the consensus sequence flanking translational start sites in Drosophila and vertebrates. Nucleic Acids Res. 15: 1353-1361.

Chesnokov, I., Gossen, M., Remus, D., and Botchan, M. 1999. Assembly of functionally active Drosophila origin recognition complex from recombinant proteins. Genes \& Dev. 13: $1289-1296$.

Chong, J.P.J., Hayashi, M.K., Simon, M.N., Xu, R.-M., and Stillman, B. 2000. A double-hexamer archael minichromosome maintenance protein is an ATP-dependent DNA helicase. Proc. Natl. Acad. Sci. 97: 1530-1535.

Clyne, R. and Kelly, T.J. 1995. Genetic analysis of an ARS element from the fission yeast Schizosaccharomyces pombe. EMBO I. 14: 6348-6357.

Coleman, T.R., Carpenter, P.B., and Dunphy, W.G. 1996. The Xenopus Cdc6 protein is essential for the initiation of a single round of DNA replication in cell-free extracts. Cell 87: 53-63.

DePamphilis, M.L. 1999. Replication origins in metazoan chromosomes: Fact or fiction. BioEssays 21: 5-16.

Donaldson, A.D. and Blow, J.J. 1999. The regulation of replication origin activation. Curr. Opin. Genet. Dev. 9: 62-68.

Donovan, S.H., Drury, L.S., and Diffley, J. 1997. Cdc6p-dependent loading of $\mathrm{Mcm}$ proteins onto pre-replicative chromatin in budding yeast. Proc. Natl. Acad. Sci. 94: 5611-5616.

Dubey, D.D., Zhu, J., Carlson, D.L., Sharma, K., and Huberman, J.A. 1994. Three ARS elements contribute to the ura4 replication origin region in the fission yeast, Schizosaccharomyces pombe. EMBO J. 13: 3638-3647.

Duronio, R.J. and O'Farrell, P.H. 1994. Developmental control of a G1-S transcriptional program in Drosophila. Development 120: 1503-1515.

- 1995. Developmental control of the $\mathrm{G}_{1}$ to $\mathrm{S}$ transition in Drosophila: cyclin E is a limiting downstream target of E2F. Genes \& Dev. 9: 1456-1468.

Duronio, R.J., O'Farrell, P.H., Xie, J.-E., Brook, A., and Dyson, N. 1995. The transcription factor E2F is required for $S$ phase during Drosophila embryogenesis. Genes \& Dev. 9: 14451455.

Duronio, R.J., Bonnette, P.C., and O'Farrell, P.H. 1998. Mutations of the Drosophila dDP, dE2F, and cyclin E genes reveal distinct roles for the E2F-DP transcription factor and cyclin E during the G1-S transition. Mol. Cell. Biol. 18: 141-151.

Dutta, A. and Bell, S.P. 1997. Initiation of DNA replication in eukaryotic cells. Annu. Rev. Cell Dev. Biol. 13: 293-332.

Feger, G., Vaessin, H., Su, T.T., Wolff, E., Jan, L.Y., and Jan, Y.N. 1995. dpa, a member of the MCM family, is required for mitotic DNA replication but not endoreplication in Drosophila. EMBO J. 14: 5387-5398.

Foe, V.E., Odell, G.M., and Edgar, B.A. 1993. Mitosis and morphogenesis in the Drosophila embryo: Point and counterpoint. In The development of Drosophila melanogaster (ed. M. Bate and A. Martinez Arias), pp. 149-300. Cold Spring Harbor Laboratory Press, Cold Spring Harbor, NY.
Gloor, G. and Engels, W. 1992. Single-fly DNA preps for PCR Drosophila Information Service 71: 148-149.

Gossen, M., Pak, D.T., Hansen, S.K., Acharya, J.K., and Botchan, M.R. 1995. A Drosophila homolog of the yeast origin recognition complex. Science 270: 1674-1677.

Hartwell, L. 1973. Three additional genes required for deoxyribonucleic acid synthesis in Saccharomyces cerevisiae. I. Bacteriol. 115: 966-974.

1978. Cell division from a genetic perspective. J. Cell Biol. 77: 627-637.

Hendzel, M.J., Wei, Y., Mancini, M.A., Van Hooser, A., Ranalli, T., Brinkley, B.R., Bazett-Jones, D.P., and Allis, C.D. 1997. Mitosis-specific phosphorylation of histone H3 initiates primarily within pericentromeric heterochromatin during G2 and spreads in an ordered fashion coincident with mitotic chromosome condensation. Chromosoma 106: 348360.

Hofmann, J.F.X. and Beach, D. 1994. cdt1 is an essential target of the Cdc10/Sct1 transcription factor: Requirement for DNA replication and inhibition of mitosis. EMBO J. 13: 425434.

Hollingsworth, R.E. and Sclafani, R.A. 1990. DNA metabolism gene CDC7 from yeast encodes a serine (threonine) protein kinase. Proc. Natl. Acad. Sci. 87: 6272-6276.

Homesley, L., Lei, M., Kawasaki, Y., Sawyer, S., Christensen, T., and Tye, B.K. 2000. Mcm10 and the MCM2-7 complex interact to initiate DNA synthesis and to release replication factors from origins. Genes \& Dev. 14: 913-926.

Ishimi, Y. 1997. A DNA helicase activity is associated with an MCM4, -6, and -7 protein complex. J. Biol. Chem. 272: 24508-24513.

Jackson, A.P., Harrison, K., Rosamond, J., and Sclafani, R.A. 1993. Cell cycle regulation of the yeast Cdc7 protein kinase by association with the Dbf4 protein. Mol. Cell. Biol. 13: 2899-2908.

Kelly, T.J., Martin, G.S., Forsburg, S.L., Stephen, R.J., Russo, A., and Nurse, P. 1993. The fission yeast $c d c 18^{+}$gene product couples S phase to START and mitosis. Cell 74:371-382.

Kelman, Z., Lee, L.E., and Hurwitz, J. 1999. The single minichromosome maintenance protein of Methanobacterium thermozutotrophicum DeltaH contains DNA helicase activity. Proc. Natl. Acad. Sci. 96: 14783-14788.

Landis, G. and Tower, J. 1999. The drosophila chiffon gene is required for chorion gene amplification, and is related to the yeast dbf4 regulator of DNA replication and cell cycle. Development 126: 4281-4293.

Landis, G., Kelley, R., Spradling, A.C., and Tower, J. 1997. The $k 43$ gene, required for chorion gene amplification and diploid cell chromosome replication, encodes the Drosophila homolog of yeast origin recognition complex subunit 2. Proc. Nat1. Acad. Sci. 94: 3888-3892.

Li, J.J. and Deshaies, R.J. 1993. Exercising self restraint: Discouraging illicit acts of S and M in eukaryotes. Cell 74: 223226.

Maiorano, D., Moreau, J., and Mechali, M. 2000. XCDT1 is required for the assembly of pre-replicative complexes in Xenopus laevis. Nature 404: 622-625.

Marahrens, Y. and Stillman, B. 1992. A yeast chromosomal origin of replication defined by multiple functional elements. Science 255: 817-823.

McCall, K., O'Connor, M.B., and Bender, W. 1994. Enhancer traps in the Drosophila bithorax complex mark parasegmental domains. Genetics 138: 387-399.

Nishitani, H., Lygerou, Z., Nishimoto, T., and Nurse, P. 2000. The Cdt1 protein is required to license DNA for replication in fission yeast. Nature 404: 625-628. 
Orr-Weaver, T.L. 1991. Drosophila chorion genes: Cracking the eggshell's secrets. BioEssays 13: 97-105.

Pak, D.T., Pflumm, M., Chesnokov, I., Huang, D.W., Kellum, R., Marr, J., Romanowski, P., and Botchan, M.R. 1997. Association of the origin recognition complex with heterochromatin and HP1 in higher eukaryotes. Cell 91: 311-323.

Pardue, M.L. 1994. Looking at polytene chromosomes. Methods Cell Biol. 44: 333-351.

Piatti, S., Lengauer, C., and Nasmyth, K. 1995. Cdc6 is an unstable protein whose de novo synthesis in G1 is important for the onset of S phase and for preventing a "reductional" anaphase in the budding yeast Saccharomyces cerevisiae. EMBO I. 14: 3788-3798.

Rechsteiner, M. and Rogers, S.W. 1996. PEST sequences and regulation of proteolysis. Trends Biochem. Sci. 21: 267-271.

Roch, F., Serras, F., Cifuentes, F.J., Corominas, M., Alsina, B., Lopez-Varea, A., Hernandez, R., Guerra, D., Caricchi, S. et al. 1998. Screening of larval/pupal P-element induced lethals on the second chromosome in Drosophila melanogaster: Clonal analysis and morphology of imaginal discs. Mol. Gen. Genet. 257: 103-112.

Royzman, I. 1998. Drosophila E2F and DP genes: Their role in the regulation of G1-S progression and the activity of DNA replication origins. Ph.D. thesis. Massachusetts Institute of Technology, Cambridge, MA.

Royzman, I. and T.L. Orr-Weaver. 1998. S phase and differential DNA replication during Drosophila oogenesis. Genes Cells 3: 767-776.

Royzman, I., Whittaker, A.J., and Orr-Weaver, T.L. 1997. Mutations in Drosophila DP and E2F distinguish G1-S progression from an associated transcriptional program. Genes \& Dev. 11: 1999-2011.

Royzman, I., Austin, R.J., Bosco, G., Bell, S.P., and Orr-Weaver, T.L. 1999. ORC localization in Drosophila follicle cells and the effects of mutations in $d E 2 F$ and $d D P$. Genes \& Dev. 13: $827-840$.

Sauer, K., Knoblich, J.A., Richardson, H., and Lehner, C.F. 1995. Distinct modes of cyclin $\mathrm{E} / \mathrm{cdc} 2 \mathrm{c}$ kinase regulation and Sphase control in mitotic and endoreduplication cycles of Drosophila embryogenesis. Genes \& Dev. 9: 1327-1339.

Schupbach, T. and Wieschaus, E. 1989. Female sterile mutations on the second chromosome of Drosophila melanogaster. I. Maternal effect mutations. Genetics 121: 101-117.

Smith, A.V. and Orr-Weaver, T.L. 1991. The regulation of the cell cycle during Drosophila embryogenesis: The transition to polyteny. Development 112: 997-1008.

Smith, A.V., King, J.A., and Orr-Weaver, T.L. 1993. Identification of genomic regions required for DNA replication during Drosophila embryogenesis. Genetics 135: 817-829.

Spradling, A.C., Stern, D.M., Kiss, I., Roote, J., Laverty, T., and Rubin, G.M. 1995. Gene disruptions using P transposable elements: An integral component of the Drosophila genome project. Proc. Nat1. Acad. Sci. 92: 10824-10830.

Su, T.T., Feger, G., and O'Farrell, P.H. 1996. Drosophila MCM protein complexes. Mol. Biol. Cell 7: 319-329.

Tanaka, T., Knapp, D., and Nasmyth, K. 1997. Loading of an $\mathrm{Mcm}$ protein onto DNA replication origins is regulated by Cdc6p and CDKs. Cell 90: 649-660.

Tang, T.T.-L., Bickel, S.E., Young, L.M., and Orr-Weaver, T.L. 1998. Maintenance of sister-chromatid cohesion at the centromere by the Drosophila MEI-S332 protein. Genes \& Dev. 12: 3843-3856.

Tautz, D. and Pfeifle, C. 1989. A non-radioactive in situ hybridization method for the localization of specific RNAs in Drosophila embryos reveals translational control of the segmentation gene hunchback. Chromosoma 98: 81-85.
Torok, T., Tick, G., Alvardo, M., and Kiss, I. 1993. P-lacW insertional mutagenesis on the second chromosome of Drosophila melanogaster: Isolation of lethals with different overgrowth phenotypes. Genetics 135: 71-80.

Treisman, J.E., Follette, P.J., O'Farrell, P.H., and Rubin, G.M. 1995. Cell proliferation and DNA replication defects in a Drosophila MCM2 mutant. Genes \& Dev. 9: 1709-1715.

Underwood, E.M., Briot, A.S., Doll, K.Z., Ludwiczak, R.L., Otteson, D.C., Tower, J., Vessey, K.B., and Yu, K. 1990. Genetics of 51D-52A, a region containing several maternal-effect genes and two maternal-specific transcripts in Drosophila. Genetics 126: 639-650.

Wolf, E., Kim, P.S., and Berger, B. 1997. MultiCoil: A program for predicting two- and three-stranded coiled coils. Protein Sci. 6: 1179-1189.

Yoon, H. and Campbell, J.L. 1991. The CDC7 protein of Saccharomyces cerevisiae is a phosphoprotein that contains protein kinase activity. Proc. Natl. Acad. Sci. 88: 3574-3578.

$\mathrm{Yu}, \mathrm{W}$. and Gibbs, R.A. 1997. Large-scale concatenation cDNA sequencing. Genome Res. 7: 353-358. 


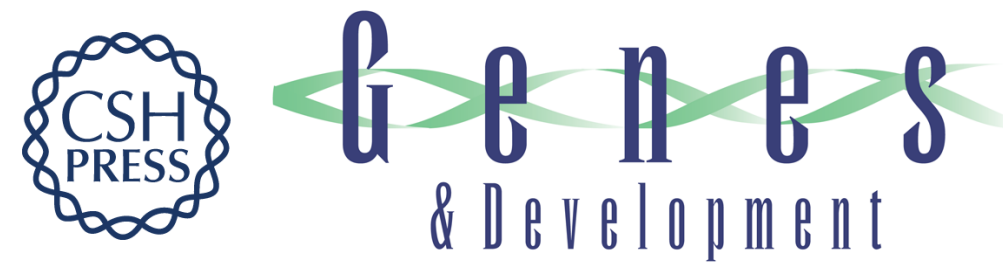

\section{Drosophila Double parked: a conserved, essential replication protein that colocalizes with the origin recognition complex and links DNA replication with mitosis and the down-regulation of $\mathbf{S}$ phase transcripts}

Allyson J. Whittaker, Irena Royzman and Terry L. Orr-Weaver

Genes Dev. 2000, 14:

Access the most recent version at doi:10.1101/gad.14.14.1765

References

This article cites 67 articles, 36 of which can be accessed free at: http://genesdev.cshlp.org/content/14/14/1765.full.html\#ref-list-1

License

Email Alerting Service

Receive free email alerts when new articles cite this article - sign up in the box at the top right corner of the article or click here.

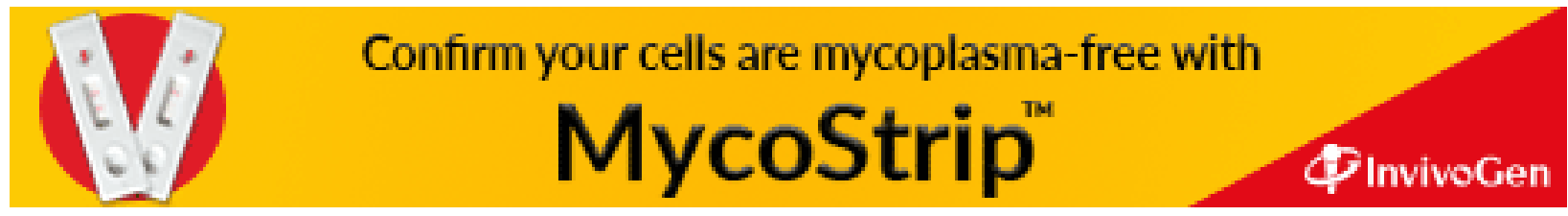

